\title{
ARTICLE OPEN \\ Laterally confined photonic crystal surface emitting laser incorporating monolayer tungsten disulfide
}

\author{
Xiaochen $\mathrm{Ge} \mathbb{D}^{1}$, Momchil Minkov ${ }^{2}$, Shanhui Fan ${ }^{2}$, Xiuling Li $\mathbb{D}^{3}$ and Weidong Zhou ${ }^{1}$
}

With an ultimately thin active region, monolayer transition metal dichalcogenide lasers have the potential of realizing ultralow lasing threshold and power consumption. The flexibility also enables integration possibilities on unconventional substrates. Here, we report a photonic crystal surface emitting laser using monolayer tungsten disulfide as the gain medium. The cavity design utilizes a heterostructure in the photonic crystal lattice to provide lateral confinement for a high quality factor with a compact active region. Room-temperature continuous wave lasing is realized after integrating monolayer tungsten disulfide flakes onto the silicon nitride photonic crystal on a quartz substrate. Highly directional, near surface-normal emission has also been experimentally demonstrated. The work reported here demonstrates that a large-area single-mode directional laser can be realized from a monolayer gain medium, which is critical for laser scaling for on-chip integration in data and sensing applications.

npj 2D Materials and Applications (2019)3:16; https://doi.org/10.1038/s41699-019-0099-1

\section{INTRODUCTION}

As a direct bandgap semiconductor, monolayer transition metal dichalcogenide (TMDC) draws tremendous research interests, and numerous monolayer or few-layer TMDC-based lasers ${ }^{1-6}$ and lightemitting diodes ${ }^{7-11}$ have emerged recently. With an ultimately thin active region, TMDC lasers have the potential of realizing ultralow lasing threshold and power consumption. The flexibility also enables integration possibilities on unconventional substrates. $^{12,13}$ The first demonstration of TMDC lasers is based on microdisk cavities ${ }^{1,3}$ and photonic crystal (PhC) defect mode microcavities. ${ }^{2}$ The emission wavelength is then extended to near infrared by introducing molybdenum ditelluride $\left(\mathrm{MoTe}_{2}\right)$ as the gain medium. ${ }^{4,6}$ While offering a low threshold and high Purcell factor, the emissions from microcavities typically have a large divergence angle, which limits their application in practical devices due to the requirement of high-numerical aperture (NA) lenses for collecting the emission. To improve the quality of the output beam toward practical applications, efforts have been made to optimize the far-field pattern of the PhC microcavity ${ }^{6}$ or by introducing distributed Bragg reflector (DBR) ${ }^{5}$ based cavities. As the PhC defect based microcavities rely on the in-plane photonic bandgap below the light line, the divergence angle is still large, even with far-field optimization. ${ }^{6}$ The DBR-based vertical cavity surface-emitting laser (VCSEL) has an intrinsically surfacenormal emission. ${ }^{5}$ However, the size of the active region and the cavity linewidth are both limited.

Based on the Fano resonance at the $\Gamma$ point of the photonic band diagram, ${ }^{14,15}$ photonic crystal surface-emitting laser (PCSEL) is a promising laser cavity design as it provides simultaneously large-area lasing and a high beam quality. ${ }^{16,17}$ In addition, low index contrast cavity is also possible due to the relaxed index contrast requirement in such cavities. Such a unique advantage motivates its implementation in various gain media and over a wide wavelength range. $^{18-23}$ However, although possessing superior optical and electrical properties than the synthesized counterpart, the achievable size and yield of exfoliated monolayer TMDC flakes are still limited, ${ }^{24}$ which makes TMDC-based PCSEL challenging. To shrink the size of the active region while maintaining the advantage of PCSEL, a lateral confinement scheme with heterostructure PhC was proposed, where modulation of the hole radii creates a mode gap near the $\Gamma$ point above the light cone. ${ }^{25}$ Here, we will present and experimentally demonstrate PCSEL with monolayer tungsten disulfide $\left(\mathrm{WS}_{2}\right)$ as the gain medium.

\section{RESULTS}

We design the PCSEL cavity in a similar fashion as in an earlier paper. ${ }^{25}$ Figure 1 a shows a sketch of the cavity design, where a hexagonal PhC lattice of air holes on a 112-nm-thick silicon nitride $\left(\mathrm{Si}_{3} \mathrm{~N}_{4}\right)$ slab on quartz, with the lattice constant $a=454 \mathrm{~nm}$, is considered. With such a low contrast of a refractive index between the $\mathrm{PhC}$ slab and the substrate, achieving vertical confinement through index guiding, and lateral confinement through a complete in-plane bandgap are both challenging. Instead, we looked into the modes above the light cone. It is well known that a symmetry-protected bound state in continuum (BIC) exists at the $\Gamma$ point with an infinite vertical quality $(Q)$ factor. ${ }^{14}$ For a finite structure, the pseudo-BIC near $\Gamma$ would provide a sufficiently high $Q$ for vertical confinement. Lateral confinement can be realized by utilizing a mode gap and the mismatch of modes at different bands. ${ }^{25}$ The PhC lattice is composed of three regions with slightly different air hole radii, as indicated by the dashed lines. The center part of the lattice, denoted as the cavity core region, containing 15 periods of air holes starting from the origin, is the active area of the laser cavity where the optical mode is localized. The lateral confinement is provided by the outer area of the PhC lattice with a reduced hole radius compared with the core region, which is denoted as the cladding. Six periods of air holes are placed between the core and cladding regions for a smooth transition of

\footnotetext{
${ }^{1}$ University of Texas at Arlington, Arlington, TX 76019, USA; ${ }^{2}$ Stanford University, Stanford, CA 94305, USA and ${ }^{3}$ University of Illinois Urbana-Champaign, Urbana, IL 61801, USA
} Correspondence: Weidong Zhou (wzhou@uta.edu)

Received: 18 December 2018 Accepted: 28 March 2019

Published online: 15 April 2019 
the hole radius, which improves the $Q$ factor of the cavity mode. More specifically, the air hole radius in each $\mathrm{PhC}$ region is modulated to be $R_{\text {core }}=0.25 a, R_{\text {trans }}=0.24 a$, and $R_{\text {clad }}=0.22 a$, respectively. The electric field intensity of the fundamental TE-like mode, which has the highest $Q$, is plotted in Fig. $1 \mathrm{~b}$ and the mode is well confined within the core region of the $\mathrm{PhC}$ cavity, even though there is an absence of a complete in-plane photonic bandgap. The theoretical resonant wavelength and $Q$ factor are determined to be $\lambda=628.3 \mathrm{~nm}$ and $Q \approx 23,500$. The high $Q$ factor is essential for realizing TMDC lasers, as the monolayer gain medium results in a fundamentally limited confinement factor, which increases the material gain requirement for achieving lasing. (a)

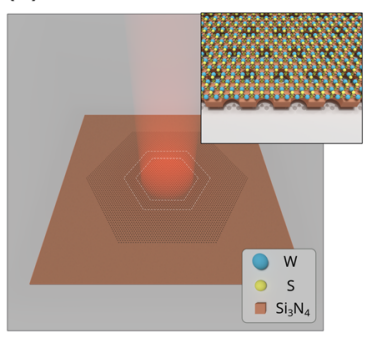

(b)

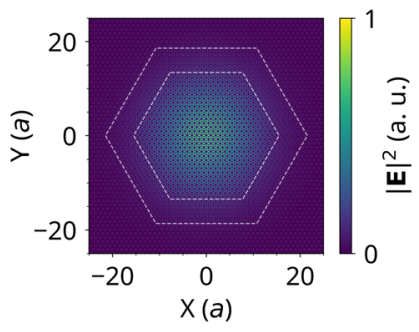

Fig. 1 Design of the $\mathrm{WS}_{2}$-based PCSEL. a Sketch of the cavity configuration and an illustration of the surface-normal emission from the active region. The inset shows a cross-sectional view of the $W_{2}$ integration scheme. b 3D FDTD simulated field intensity of the fundamental cavity mode near the cavity core region. In both subfigures, white dashed lines are drawn at the interfaces of each $\mathrm{PhC}$ region as a visual guide
As the PhC cavity mode is near the $\Gamma$ point in the band diagram, directional surface-normal emission would be expected, as illustrated in Fig. 1a. The inset of Fig. 1a shows the zoomed-in cross-sectional view of the schematic of monolayer $\mathrm{WS}_{2}$ integration, where the flake is directly transferred onto the $\mathrm{Si}_{3} \mathrm{~N}_{4} \mathrm{PhC}$, which can be done by the widely used transfer-printing processes. $^{26}$

We fabricated the PhC cavities on silicon nitride to verify the design. Figure 2a shows a scanning electron microscopy (SEM) image of a representative cavity sample near the core region, where the boundaries between adjacent regions are indicated by dashed lines. The resonances of the fabricated devices were characterized by photoluminescence $(\mathrm{PL})$, utilizing the cavityenhanced intrinsic fluorescence of silicon nitride. ${ }^{27}$ Plotted in Fig. $2 \mathrm{~b}$ and fitted with a Lorentzian function, the measured passive resonance before transferring $W_{2}$ shows a linewidth of $\Delta \lambda=$ $0.13 \mathrm{~nm}$, corresponding to $Q \approx 5000$. The deviation between the simulated and measured $Q$ factors may be attributed to the nonuniformity and imperfections, which are inevitable in fabrication, and contribute to the vertical leakage and scattering loss.

After identifying the device with a high $Q$ resonance in the wavelength range of interest, monolayer $\mathrm{WS}_{2}$ flakes were prepared and transferred onto the sample. The details can be found in the Methods section and the supplementary information. Figure $2 \mathrm{~b}$ shows the microscopy image of the fabricated device, where the PhC cavity is completely covered by monolayer $\mathrm{WS}_{2}$. The completed devices were characterized in room temperature. The measured PL spectra at a pump power density of $4 \mathrm{~kW} / \mathrm{cm}^{2}$ are plotted in Fig. $2 \mathrm{~d}$. The on-cavity spectrum was collected at the center of the cavity core region and the off-cavity spectrum was collected from the same monolayer $\mathrm{WS}_{2}$ flake but outside of the $\mathrm{PhC}$ area. A sharp resonant peak corresponding to the fundamental cavity mode can be observed at $637 \mathrm{~nm}$, with the fitted full (a)

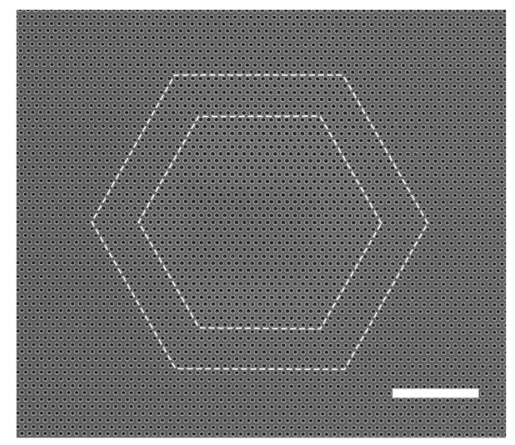

(c)

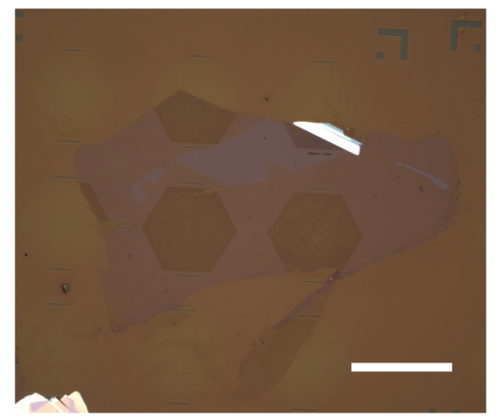

(b)

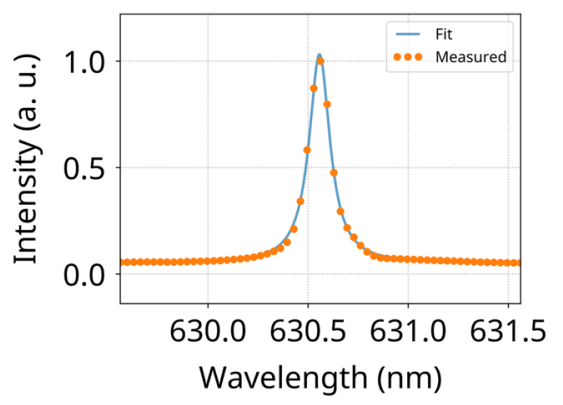

(d)

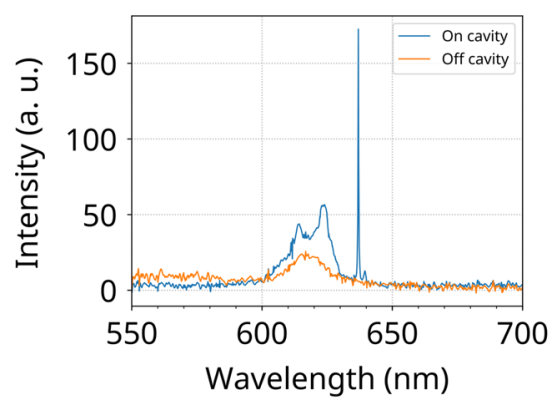

Fig. 2 Experimental realization of the WS $\mathrm{WS}_{2}$ PCSEL. a SEM image of a fabricated $\mathrm{Si}_{3} \mathrm{~N}_{4}$ cavity without $\mathrm{WS}_{2}$ (scale bar: $5 \mu \mathrm{m}$ ). The dashed lines separate $\mathrm{PhC}$ regions with different air hole radii. b Measured passive cavity resonance before transferring the monolayer $\mathrm{WS}_{2}$ flake. $\mathbf{c}$ Microscopy image of a WS $S_{2}$ flake transferred onto the $\mathrm{Si}_{3} \mathrm{~N}_{4} \mathrm{PhC}$ cavity (scale bar: $50 \mu \mathrm{m}$ ). d Measured PL spectra at $4.0 \mathrm{~kW} / \mathrm{cm}^{2}$ pump power density 
(a)

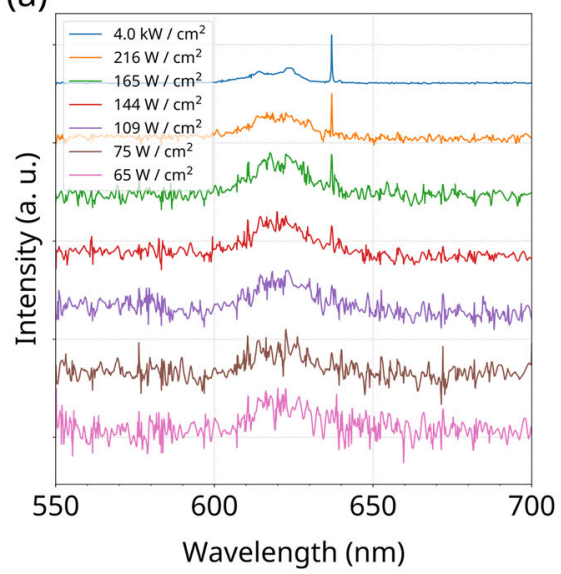

(b)

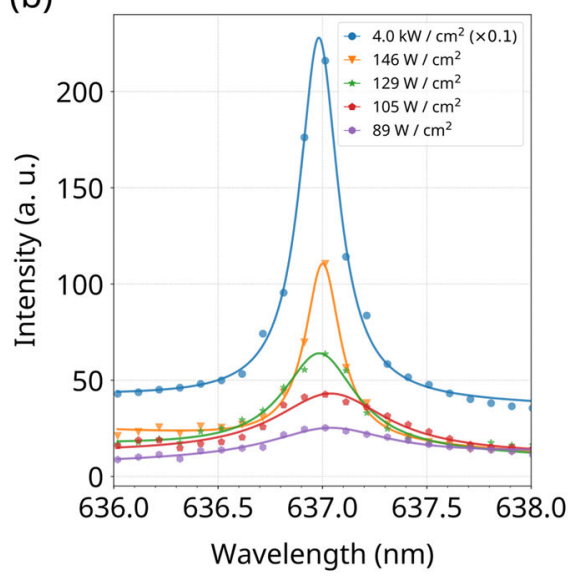

(d)

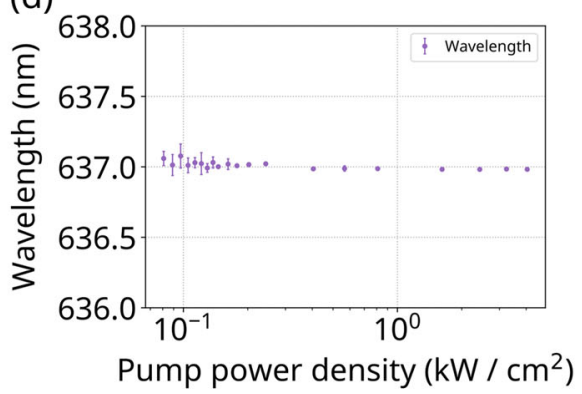

Fig. 3 Characterization of the WS 2 PCSEL. a Normalized power-dependent PL spectra. The curves are shifted in the vertical direction for clarity. b Lorentzian fitting of the cavity resonance peak near and above the threshold. c L-L curve of the resonant peak intensity and the corresponding fitted linewidths. e Power dependent resonant wavelengths

width at half-maximum linewidth of $0.25 \mathrm{~nm}(Q \approx 2500)$. The slight redshift of the lasing resonant wavelength compared with the passive device is due to the presence of the monolayer $\mathrm{WS}_{2}$ and the protective encapsulation. In the supplementary information, it is shown that for the pseudo-BIC that the cavity mode is based on, the observed redshift is reasonable. Apart from the dominating cavity peak, a few broad resonant features can be seen by modulating the $\mathrm{WS}_{2}$ exciton peak centered around $620 \mathrm{~nm}$. Those features are from higher-order bulk PhC modes that are not confined by the PhC mode gap. The enhancement from the bulk modes, together with the suspension of monolayer $\mathrm{WS}_{2}$ over the air holes, contributes to the overall enhancement of the on-cavity $\mathrm{WS}_{2}$ exciton emission compared with the off-cavity case.

To study the transition from spontaneous emission to lasing, we performed power dependent PL measurement on the device. In the measured PL spectra at lower pump power in Fig. 3a, only the broad PL from the spontaneous emission of $\mathrm{WS}_{2}$ is observed. With the increasing pump power, a resonant peak at $637 \mathrm{~nm}$ emerges and eventually outgrows the background spontaneous emission. We fitted the peak with a Lorentzian function, as shown in Fig. 3b, to obtain the peak intensities and linewidths. As the cavity resonance peak cannot be clearly defined due to the noise and fluctuation below the pump power density of $89 \mathrm{~W} / \mathrm{cm}^{2}$, we fitted the background PL peak for the linewidth below the threshold. In the light-in, light-out $(\mathrm{L}-\mathrm{L})$ curve in Fig. $3 \mathrm{c}$, a soft transition region can be observed near $100 \mathrm{~W} / \mathrm{cm}^{2}$, where the peak with a linewidth near $0.7 \mathrm{~nm}$ emerges and grows. The linewidth narrows down to $0.2 \sim 0.3 \mathrm{~nm}$ above $144 \mathrm{~W} / \mathrm{cm}^{2}$ of pump power density. The region between 89 and $144 \mathrm{~W} / \mathrm{cm}^{2}$ is shaded in gray in Fig. 3c, coinciding with the change of the slope in the $L-L$ curve, which indicates that the device transits from amplified spontaneous emission to lasing. Meanwhile, the wavelength stays nearly constant above the (a)

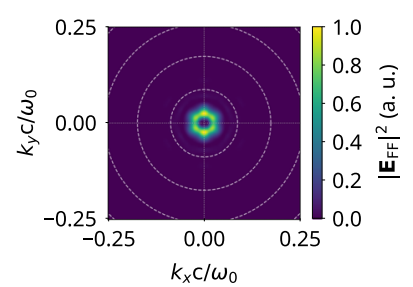

(b)

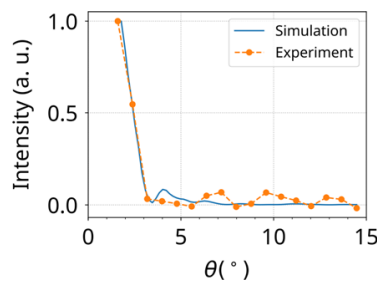

Fig. 4 Emission directionality of the $\mathrm{WS}_{2}$ PCSEL. a FDTD simulated far-field radiation pattern. The dashed circles are the collection angle $\theta$ in $5^{\circ}$ steps. b Comparison between simulated and measured emission profile depending on $\theta$. The minimum angle is $\theta_{\min }=1.59^{\circ}$ for both cases

threshold, as shown in Fig. 3d, which can be understood as thermal effect was not observed in the range of pump power.

As the cavity design is based on PCSEL, surface-normal emission in the far field can be expected. The FDTD simulated far-field emission profile of the lasing mode is plotted in Fig. 4a. Indeed, the emission is concentrated near surface normal within a few degrees. To verify that experimentally, the angle of collection $\theta$ was controlled by varying the diameter of an iris diaphragm in the infinity space of the setup. The angle dependence of the far-field profile of the cavity resonance is plotted in Fig. $4 \mathrm{~b}$. The simulated emission profile is obtained from Fig. 4 a by extracting the intensity depending on $\theta$ while matching the range of $\theta$ obtainable in the measurement. The measured and simulated profiles agree well within the range of $\theta$ measured, indicating that the laser emission from the $\mathrm{PhC}$ cavity is concentrated within $\pm 3^{\circ}$ of the surfacenormal direction. 


\section{DISCUSSION}

In agreement with previous reports, ${ }^{1,5}$ we observed lasing on the longer-wavelength side of the monolayer $\mathrm{WS}_{2} \mathrm{PL}$ spectrum. Multiple batches of samples were prepared with the passive high $Q$ resonances tuned over the wavelength range of the PL peak of monolayer $\mathrm{WS}_{2}$. After integration of $\mathrm{WS}_{2}$, all the devices showing a signature of lasing have the wavelengths near $640 \mathrm{~nm}$. As shown in the supplementary information, the devices resonating at shorter wavelengths only show PL enhancement with linewidths near $1 \mathrm{~nm}$, despite having similar passive $Q$ factors as the lasing devices. The reabsorption of $\mathrm{WS}_{2}$ makes the material show loss instead of gain at shorter wavelengths. Therefore, to achieve lasing, it is critical to have a high $Q$ resonance matching the gain wavelengths. In a recent report, ${ }^{28}$ it is also shown theoretically that the gain of monolayer $\mathrm{WS}_{2}$ would indeed emerge from the long-wavelength side. With an estimated confinement factor of $\Gamma_{\text {Ws2 }}=0.092 \%$, we have obtained that $\Gamma_{\text {wS2 }} Q=2.3$, which exceeds the $\mathrm{WS}_{2}$ material-specific value of 2.18 which is required for the gain to compensate the cavity loss. ${ }^{28}$ On the other hand, the quantum threshold condition, the mean photon number exceeding unity, was suggested as a criterion of lasing in TMDC microcavity emitters, ${ }^{29,30}$ which the presented device, together like the previous reports, ${ }^{1-5}$ does not satisfy.

In summary, we have demonstrated a room-temperature heterostructure photonic crystal surface-emitting laser using monolayer $\mathrm{WS}_{2}$ as the gain medium. Compared with previous reports, $^{1-6}$ the presented cavity has a larger active region. A linewidth narrowing with a factor over 2 is observed, which suggests that one of the lasing condition has been met. ${ }^{29}$ The lasing mode shows a highly directional emission within $\pm 3^{\circ}$ from the experimentally measured far-field profile. While a monolayer gain medium has the potential in achieving a low threshold due to minimizing the power requirement for achieving gain, it also imposes a fundamental limit on the output power of the light source, which will be an issue for any practical application. Challenges also come from the instability of the TMDC material, such as sensitivity to the environment, ${ }^{31,32}$ aging, and degradation issues. ${ }^{33,34}$ While forming a multi-quantum-well-like structure by stacking multiple isolated monolayers is a viable approach for overcoming the output power limit, we believe that the investigation on the scalable PCSEL platform and the engineering of light extraction, together with the progresses on the synthesized, large-area monolayers, would also be promising in realizing low power consumption, high efficiency semiconductor lasers.

\section{METHODS}

\section{Numerical simulation}

The cavity resonances are simulated by 3D finite-difference time-domain (FDTD) method. ${ }^{35}$ Due to the scale of the problem, the resolution is 20 grids per lattice constant, and the presence of the monolayer $\mathrm{WS}_{2}$ and CYTOP was not considered. In the supplementary information, rigorous coupled wave analysis (RCWA) method ${ }^{36}$ is used to simulate the redshift of the pseudo-BIC caused by monolayer $\mathrm{WS}_{2}$ and CYTOP. The far-field radiation pattern is calculated through a near-to-far-field transformation ${ }^{37}$ after obtaining the near field through FDTD.

\section{PhC cavity fabrication}

Stoichiometric $\mathrm{Si}_{3} \mathrm{~N}_{4}$ was deposited on a quartz substrate by low-pressure chemical vapor deposition (LPCVD). The PhC cavities were patterned on ZEP520A e-beam resist by electron beam lithography (EBL). Prior to the EBL process, a thin layer of conductive polymer (Allresist AR-PC 5090) was coated on top of the resist for charge dissipation. The pattern was then transferred to $\mathrm{Si}_{3} \mathrm{~N}_{4}$ by inductively coupled plasma reactive ion etching (ICP-RIE) using a gas combination of $\mathrm{SF}_{6}, \mathrm{CHF}_{3}$, and helium. After stripping the resist in hot $\mathrm{N}$-methyl-2-pyrrolidone, the sample was cleaned in a gentle oxygen plasma RIE process to remove the residues.

\section{Monolayer $\mathrm{WS}_{2}$ preparation and integration}

Monolayer $\mathrm{WS}_{2}$ was prepared by a gold-assisted exfoliation process. ${ }^{24}$ Bulk $W_{2}$ was first dispensed on tape. A layer of gold $(100 \mathrm{~nm})$ was deposited on $W S_{2}$ by electron beam evaporation. To exfoliate $W S_{2}$ flakes from bulk crystals, a polydimethylsiloxane (PDMS) stamp attached to a glass slide was used. Owing to the affinity between sulfur and gold, ${ }^{24,38}$ large monolayer flakes can be obtained, which show a profound color contrast under optical microscope inspection. After being identified, the gold-coated $\mathrm{WS}_{2}$ flakes were transfer-printed onto a silicon oxide substrate using an automatic assembly platform (FiconTEC FL-200). The $\mathrm{SiO}_{2}$ surface was then cleaned by oxygen plasma for activation and contamination removal. The gold layer was removed in $\mathrm{KI} / \mathrm{I}_{2}$ solution to expose the $\mathrm{WS}_{2}$ flakes.

To transfer the $\mathrm{WS}_{2}$ flakes onto $\mathrm{Si}_{3} \mathrm{~N}_{4} \mathrm{PhCs}$, a layer of poly(methyl methacrylate) (PMMA) was coated on the $\mathrm{SiO}_{2}$ as a carrier and another piece of PDMS stamp was attached to the PMMA to provide mechanical support. The whole stack was submerged in deionized water. After applying ultrasonication, PMMA and $\mathrm{SiO}_{2}$ were separated due to water penetration, and the $\mathrm{WS}_{2}$ flakes were also released from $\mathrm{SiO}_{2}$ in the process. The $\mathrm{WS}_{2}$ flakes, together with the PMMA carrier, were aligned and transfer-printed onto the target $\mathrm{Si}_{3} \mathrm{~N}_{4} \mathrm{PhC}$ cavities. With the support of the PMMA film, the monolayer flakes can be completely transferred onto the patterned substrate. The PMMA carrier was then removed in acetone. Finally, a thin layer of a low refractive index fluoropolymer (CTL-809M from AGC Chemicals diluted to $1 \%$ ) was spun-coated on top of the $\mathrm{WS}_{2}$ for protection. $^{34}$

Microscopy images of the steps during the preparation and transfer procedures can be found in the supplementary information.

\section{Characterization}

The fabricated sample was characterized on a home-built microphotoluminescence ( $\mu$-PL) setup sketched in the supplementary information. A CW 450-nm laser diode with a pigtailed single mode fiber was used to pump the device. The light from the single mode fiber was collimated by a fiber collimator, expanded by a telescope, and focused onto the center of the cavity by a 0.25 NA objective lens (Olympus PLN 10x). The pump spot size was calculated from the camera image of the spot on an unpatterned area, with the resulting beam widths of $d_{\sigma x}=1.13 \mu \mathrm{m}$ and $d_{o y}=1.04 \mu \mathrm{m}$. The PL signal was collected by the same objective lens and directed to a monochromator (Horiba iHR-550) with a cooled chargecoupled device (CCD) camera (Horiba Synapse BIUV) for spectrum acquisition. To collect the weak emission signal at low pump power, pixel binning and a long exposure time (30-120 s) were employed.

A variable iris diaphragm was placed at the momentum space to restrict the angle of collection. Prior to the actual measurement, the collection light path was aligned such that the emission normal to the sample surface would pass through the center of the iris diaphragm. The angle of collection $\theta$ can be calculated from $\theta(D) \approx \arcsin \left(D / 2 f_{\text {obj }}\right)$, where $f_{\text {obj }}=$ $18 \mathrm{~mm}$ is the focal length of the objective lens and $D$ is the diameter of the iris. Limited by the minimum diameter of the iris diaphragm and the NA of the objective lens, $D$ was changed from $D_{\min }=1.0 \mathrm{~mm}$ to $D_{\max }=9.0 \mathrm{~mm}$ in $0.5 \mathrm{~mm}$ steps, corresponding to the $\theta$ range from $1.59^{\circ}$ to $14.48^{\circ}$. At each $\theta$ angle, the PL spectrum of the cavity pumped above the threshold was measured, and the lasing peak height was extracted by Lorentzian fitting. The angle-dependent emission profile can thus be reconstructed by performing a central difference of the accumulated peak heights in respect to the angle $\theta^{39}$

\section{DATA AVAILABILITY}

The data that support the findings of this study are available from the corresponding author upon reasonable request.

\section{ACKNOWLEDGEMENTS}

This work is supported by Air Force Office of Scientific Research Grant No. FA9550-161-0010 (PM: Dr. Gernot Pomrenke). M.M. acknowledges the funding through the Swiss National Science Foundation project number P300P2_177721. X.G. thanks Dr. Galan Moody for the helpful discussions.

\section{AUTHOR CONTRIBUTIONS}

W.Z., S.F., and X.L. conceived the project. M.M. and X.G. designed the cavity and performed simulation. X.G. prepared and characterized the samples. X.G. and W.Z. 
analyzed the PL data. All authors discussed on the results and commented on the paper.

\section{ADDITIONAL INFORMATION}

Supplementary information accompanies the paper on the npj $2 D$ Materials and Applications website (https://doi.org/10.1038/s41699-019-0099-1).

Competing interests: The authors declare no competing interests.

Publisher's note: Springer Nature remains neutral with regard to jurisdictional claims in published maps and institutional affiliations.

\section{REFERENCES}

1. Ye, Y. et al. Monolayer excitonic laser. Nat. Photon. 9, 733-733 (2015).

2. Wu, S. et al. Monolayer semiconductor nanocavity lasers with ultralow thresholds Nature 520, 69-69 (2015).

3. Salehzadeh, O., Djavid, M., Tran, N. H., Shih, I. \& Mi, Z. Optically pumped two dimensional $\mathrm{MoS}_{2}$ lasers operating at room-temperature. Nano Lett. 15, 5302-5306 (2015).

4. Li, Y. et al. Room-temperature continuous-wave lasing from monolayer molybdenum ditelluride integrated with a silicon nanobeam cavity. Nat. Nanotech. 12, 987-987 (2017)

5. Shang, J. et al. Room-temperature $2 \mathrm{D}$ semiconductor activated vertical-cavity surface-emitting lasers. Nat. Commun. 8, 543-543 (2017).

6. Fang, $\mathrm{H}$. et al. $1305 \mathrm{~nm}$ few-layer $\mathrm{MoTe}_{2}$-on-silicon laser-like emission. Laser Photon. Rev. 18, 1800015-1800015 (2018).

7. Withers, F. et al. Light-emitting diodes by band-structure engineering in van der Waals heterostructures. Nat. Mater. 14, 301-301 (2015)

8. Liu, C.-H. et al. Nanocavity integrated van der Waals heterostructure lightemitting tunneling diode. Nano Lett. 17, 200-205 (2016).

9. Yang, W. et al. Electrically tunable valley-light emitting diode (vLED) based on CVD-grown monolayer WS . Nano Lett. 16, 1560-1567 (2016).

10. Bie, Y.-Q. et al. A MoTe 2 -based light-emitting diode and photodetector for silicon photonic integrated circuits. Nat. Nanotech. 12, 1124-1124 (2017).

11. Lien, D.-H. et al. A. Large-area and bright pulsed electroluminescence in monolayer semiconductors. Nat. Commun. 9, 1229-1229 (2018).

12. Lee, G.-H. et al. Flexible and transparent $\mathrm{MoS}_{2}$ field-effect transistors on hexagonal boron nitride-graphene heterostructures. ACS Nano 7, 7931-7936 (2013).

13. De Fazio, D. et al. High responsivity, large-area graphene/ $\mathrm{MoS}_{2}$ flexible photodetectors. ACS Nano 10, 8252-8262 (2016).

14. Fan, S. \& Joannopoulos, J. D. Analysis of guided resonances in photonic crystal slabs. Phys. Rev. B 65, 235112-235112 (2002).

15. Zhou, W. et al. Progress in 2D photonic crystal Fano resonance photonics. Prog Quant. Electron. 38, 1-74 (2014).

16. Hirose, K. et al. Watt-class high-power, high-beam-quality photonic-crystal lasers. Nat. Photon. 8, 406-411 (2014).

17. Noda, S., Kitamura, K., Okino, T., Yasuda, D. \& Tanaka, Y. Photonic-crystal Surfaceemitting Lasers: review and Introduction of Modulated-Photonic Crystals. IEEE J. Sel. Top. Quant. Electron. 23, 1-7 (2017).

18. Chua, S.-L., Chong, Y., Stone, A. D., Soljačić, M. \& Bravo-Abad, J. Low-threshold lasing action in photonic crystal slabs enabled by Fano resonances. Opt. Express 19, 1539-1562 (2011)

19. Baumann, K. et al. Organic mixed-order photonic crystal lasers with ultrasmall footprint. Appl. Phys. Lett. 91, 171108-171108 (2007).

20. Zhao, D. et al. Printed large-area single-mode photonic crystal bandedge surfaceemitting lasers on silicon. Sci. Report. 6, 18860-18860 (2016).
21. Liu, S.-C. et al. Photonic crystal bandedge membrane lasers on silicon. Appl. Opt. 56. H67-H73 (2017).

22. Kodigala, A. et al. Lasing action from photonic bound states in continuum. Nature 541, 196-199 (2017).

23. Matsubara, $\mathrm{H}$. et al. GaN photonic-crystal surface-emitting laser at blue-violet wavelengths. Science 319, 445-447 (2008).

24. Desai, S. B. et al. Gold-mediated exfoliation of ultralarge optoelectronicallyperfect monolayers. Adv. Mater. 28, 4053-4058 (2016).

25. Ge, X., Minkov, M., Fan, S., Li, X. \& Zhou, W. Low index contrast heterostructure photonic crystal cavities with high quality factors and vertical radiation coupling Appl. Phys. Lett. 112, 141105-141105 (2018).

26. Castellanos-Gomez, A. et al. Deterministic transfer of two-dimensional materials by all-dry viscoelastic stamping. 2D Mater. 1, 011002-011002 (2014).

27. Khan, M., Babinec, T., McCutcheon, M. W., Deotare, P. \& Lončar, M. Fabrication and characterization of high-quality-factor silicon nitride nanobeam cavities. Opt. Lett. 36, 421-423 (2011).

28. Lohof, F. et al. Prospects and limitations of transition metal dichalcogenide laser gain materials. Nano Lett. 19, 210-217 (2018).

29. Reeves, L., Wang, Y. \& Krauss, T. F. 2D material microcavity light emitters: to lase or not to lase? Adv. Opt. Mater. 6, 1800272-1800272 (2018).

30. Javerzac-Galy, C. et al. Excitonic emission of monolayer semiconductors near-field coupled to high-Q microresonators. Nano Lett. 18, 3138-3146 (2018).

31. Liu, B. et al. Engineering bandgaps of monolayer $\mathrm{MoS}_{2}$ and $\mathrm{WS}_{2}$ on fluoropolymer substrates by electrostatically tuned many-body effects. Adv. Mater. 28 6457-6464 (2016).

32. Yu, Y. et al. Engineering substrate interactions for high luminescence efficiency of transition-metal dichalcogenide monolayers. Adv. Funct. Mater. 26, 4733-4739 (2016).

33. Gao, J. et al. Aging of transition metal dichalcogenide monolayers. ACS Nano 10, 2628-2635 (2016).

34. Kim, H., Lien, D.-H., Amani, M., Ager, J. W. \& Javey, A. Highly stable near-unity photoluminescence yield in monolayer $\mathrm{MoS}_{2}$ by fluoropolymer encapsulation and superacid treatment. ACS Nano 11, 5179-5185 (2017).

35. Oskooi, A. F. et al. MEEP: A flexible free-software package for electromagnetic simulations by the FDTD method. Comp. Phys. Commun. 181, 687-702 (2010).

36. Liu, V. \& Fan, S. S. A free electromagnetic solver for layered periodic structures. Comp. Phys. Commun. 183, 2233-2244 (2012).

37. Vučković, J., Lončar, M., Mabuchi, H. \& Scherer, A. Optimization of the Q factor in photonic crystal microcavities. IEEE J. Quant. Electron. 38, 850-856 (2002).

38. Magda, G. Z. et al. Exfoliation of large-area transition metal chalcogenide single layers. Sci. Rep. 5, 14714-14714 (2015).

39. Nazirizadeh, Y. et al. Optical characterization of photonic crystal slabs using orthogonally oriented polarization filters. Opt. Express 16, 7153-7160 (2008)

Open Access This article is licensed under a Creative Common Attribution 4.0 International License, which permits use, sharing, adaptation, distribution and reproduction in any medium or format, as long as you give appropriate credit to the original author(s) and the source, provide a link to the Creative Commons license, and indicate if changes were made. The images or other third party material in this article are included in the article's Creative Commons license, unless indicated otherwise in a credit line to the material. If material is not included in the article's Creative Commons license and your intended use is not permitted by statutory regulation or exceeds the permitted use, you will need to obtain permission directly from the copyright holder. To view a copy of this license, visit http://creativecommons. org/licenses/by/4.0/.

(c) The Author(s) 2019 